\title{
The Role of Oxidative Stress in Cancer
}

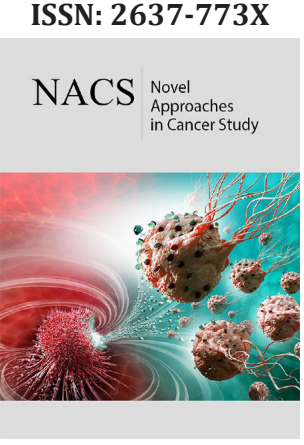

*Corresponding author: Yi Lu, Department of Pathology and Laboratory Medicine, University of Tennessee Health Science Center, Cancer Research Building, Room 258, 19 South Manassas Street, Memphis, TN 38163, USA, Email: ylu@uthsc.edu

Submission: 眥 March 10, 2020

Published: 梅 March 19, 2020

Volume 4 - Issue 2

How to cite this article: Krishna Dasari, Chikezie O Madu, Yi Lu. The Role of Oxidative Stress in Cancer. Nov Appro in Can Study.4(2). NACS.000585.2020.

DOI: 10.31031/NACS.2020.04.000585

Copyright@ Yi Lu. This article is distributed under the terms of the Creative Commons Attribution 4.0 International License, which permits unrestricted use and redistribution provided that the original author and source are credited.
Krishna Dasari', Chikezie $0 \mathrm{Madu}^{2}$ and Yi Lü ${ }^{3 *}$

${ }^{1}$ Departments of Biology and Advanced Placement Biology, White Station High School, Memphis, TN, USA, Email: kdasari2002@gmail.com, USA

${ }^{2}$ Departments of Biology and Advanced Placement Biology, White Station High School, Memphis, TN, USA, Email: maduco@scsk12.org, USA

${ }^{3}$ Department of Pathology and Laboratory Medicine, University of Tennessee Health Science Center, Memphis, TN, USA, Email: ylu@uthsc.edu, USA

\begin{abstract}
Oxidative stress has increasingly been considered a factor in numerous diseases and cancers because of its extensive presence throughout the body. Reactive Oxygen Species (ROS), generated continuously from oxidative phosphorylation and received from various exogenous sources, are constantly used as a key molecule for signal transduction, but under conditions of oxidative stress, when they can no longer be suppressed, they have the potential to damage the cell's DNA. In many cases, repair mechanisms mitigate the damage, and if the damage becomes irreparable, then the cell will undergo apoptosis. In rare cases, though, DNA can get damaged to a point where the cell becomes cancerous and can eventually form a tumor. While ROS often take a significant amount of time to accumulate, once they have done so and are unable to be suppressed by cellular countermeasures, it is very likely for DNA damage to occur, and in cancerous cells, they actually enhance rapid proliferation, chronic inflammation, metastasis, and angiogenesis. This is why it is necessary to boost or introduce cellular countermeasures to mitigate the dangers of ROS. Current research on oxidative stress is mainly focusing on the benefits of antioxidants, which are commonly used by the cell to reduce ROS, and triggering apoptosis in tumors through enhanced oxidative stress conditions. This review will discuss the role of oxidative stress in tumorigenesis and cancer proliferation as well as the possible treatments, especially antioxidants and ROS-inducing drugs.
\end{abstract}

\section{Introduction}

Oxidative stress refers to an often-dangerous disproportion between reactive oxygen species and the antioxidants that suppress them. The ROS mainly include superoxide anions $\left(\mathrm{O}_{2}^{-}\right.$), hydrogen peroxide $\left(\mathrm{H}_{2} \mathrm{O}_{2}\right)$, and hydroxyl radicals $(\mathrm{OH} \cdot)$. In the electron transport chain portion of cellular respiration in the mitochondria, oxygen is used as an electron acceptor. By accepting the electrons, the oxygen molecules are reduced. Thus, superoxide anions are naturally produced as a byproduct of cellular respiration. The Nox family of NADPH oxidases (which catalyzes the transportation of the electron from NADPH to oxygen) are associated with this ROS generation. Hydrogen peroxide and hydroxyl radicals are formed from subsequent transformations of this molecule. Hydroxyl radicals can also be produced as a result of the Fenton reaction in which iron donates an electron to hydrogen peroxide which is then split into two hydroxyl radicals. ROS can also be produced exogenously through radiolysis. Ionizing radiation may oxidize water, making it reactive and converting it to a hydroxyl radical [1]. As a result of being reduced, these ROS seek to oxidize other molecules in the cell, a potentially fatal action. However, despite these negative effects, ROS have some necessary functions within the cell when it is not under oxidative stress. One of their more notable roles is that of signal transduction. Within cells, ROS can oxidize cysteine residues to regulate enzyme activity, and by doing so it is involved in a number of signal transduction pathways, including the cell's response to growth factors [2].

Additionally, they play a role in the cell cycle. ROS may be implicated in the reentry of the cell in the cell cycle from the G0 phase, and the levels of ROS normally peak during the G2/M phase [3]. Lastly, the ROS play a key role in the mysterious process of memory formation through the demethylation of DNA [4]. Thus, ROS production is a necessity for organisms. However, under conditions of oxidative stress, these ROS can have terrible effects on the cell by oxidizing several components and most importantly, DNA. This significantly increases the chance for the cell to become cancerous, and this effect will be further explained in the review.

The cell utilizes antioxidants to regulate ROS levels. There are both enzymatic antioxidants and non-enzymatic antioxidants. For example, the enzyme superoxide dismutase is necessary 
for the conversion of superoxide anions into hydrogen peroxide which can then be converted into water and oxygen by catalase. The non-enzymatic antioxidant GSH reduces hydrogen peroxide to water and oxygen as well [1]. Several antioxidants are used by the cell for the conversion of various ROS into non-toxic forms, and this is why they have been looked to as a potential solution for oxidative stress problem. The other potential solution utilizes the naturally high levels of ROS in cancerous cells to selectively trigger mass apoptosis in tumors.

\section{ROS and DNA Damage}

ROS have a variety of negative effects in the cell, but the one most associated with carcinogenesis is DNA damage. Oxidation of the DNA by ROS is a major factor in this carcinogenesis because of the likelihood for it to produce mutations. These mutations are most dangerous if they cause gain-of-function in oncogenes, making the cell cancerous, or alter tumor suppressant genes like p53, inhibiting the ability of the cell to suppress carcinogenesis. Specifically, ROSassociated mutations tend to involve the transversion of guanine to thymine in the DNA [5]. This occurs because of a non-bulky mutation in which 8-oxoguanine is mispaired with adenine, and it is most often repaired by the enzyme DNA glycosylase OGG1. This also happens to occur most frequently among other mutations in the p53 gene, a key tumor suppressant [6]. However, they are not limited to just this mutation. In fact, ROS can cause several potentially carcinogenic mutations such as the oxidation of all 4 nucleotide bases, single stranded and double stranded breaks, mutations in alkali labile sites which can later form strand breaks, and general instability in DNA [7]. While deletions and translocations can occur because of ROS, substitutions are much more likely [7]. The flowchart in Figure 1 briefly summarizes the more dangerous effects of ROS in oxidatively stressed cells.

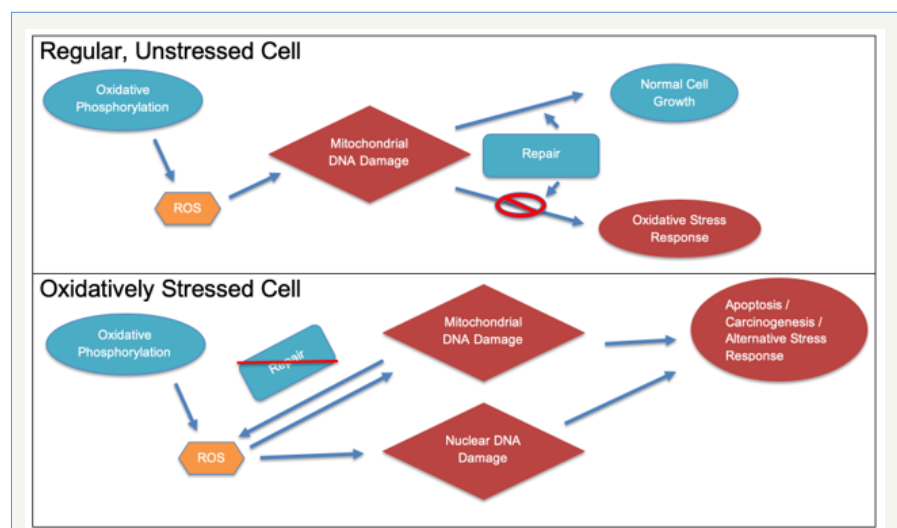

Figure 1: Different effects of ROS in a normal vs. oxidatively stressed cell. Note that in the normal cell, the effects of ROS are quickly repaired, but that is not so in a stressed cell.

A notable way to detect the excessive levels of ROS and thus oxidative stress leading to carcinogenesis is the monitoring of the 8-OHdG (8-hydroxydeozyguanosine) levels, which is the product from a hydrolysis reaction involving 8-OH-G (8-hydroxyguanosine), a molecule produced after hydroxyl-radical-associated DNA damage [8]. Importantly, 8-OHdG levels are between 8 and 17 times higher in primary breast tumors than normal [8]. While discussing the effects of free radicals on DNA, it is important to note the differences each possess. The hydroxyl radical, which forms $8-\mathrm{OHdG}$, is extremely reactive because of its unstable electron arrangement, so it reacts with most cellular components very easily. While still very dangerous because of its high reactivity, the hydroxyl radical rarely (comparative to hydrogen peroxide) is able to enter the nucleus and damage DNA. However, it still is the main contributor to ROSrelated cell damage [9]. On the other hand, hydrogen peroxide, even though it occurs in smaller concentrations than many other ROS (because of the various methods of ROS production as well as peroxisomes), is able to contribute a significant portion of the DNA damage because it is less reactive than other ROS, allowing it to diffuse through membranes and directly damage the DNA [10].

One notable example of how ROS may lead to tumorigenesis is through the accumulation of p62. In autophagy-deficient cells, which may not be able to destroy defective mitochondria, ROS are likely to accumulate. In turn, they can cause p62 accumulation, which normally triggers a DNA damage response, but under conditions of oxidative stress p62 accumulation promotes tumorigenesis [11]. Another example is how ROS can inhibit expression of the mismatch repair genes mutS homologue 2 and mutS homologue 6, impairing the ability of the cell to correct mutations and contributing to carcinogenesis [9]. Not only that, but ROS can promote the expression of methyltransferases which results in the methylation of many genes necessary for various roles like cell cycle regulation (CDKN-2), DNA repair (hMLH1), and tumor suppressants (BRCA1) [12]. Finally, referring back to the production of 8-OH-G and 8-OHdG, hydroxyl radicals can produce 8-OH-G in transcription factor binding sites because they contain many GC-rich sequences, and GC pairs are commonly targeted by free radicals. When these are damaged, the binding of transcription factors can be changed or inhibited, leading to impaired expression [1].

To date, several cancers have been linked to damage caused by ROS including breast cancer, brain cancer, prostate cancer, leukemia, lung cancer, lymphoma, sarcoma, and many more [9]. This is due to the capacity for ROS to attack and damage proteins, lipids, DNA, and be relatively unspecific in target, roaming with little resistance under conditions of oxidative stress.

\section{ROS and Inflammation, Proliferation, Metastasis, and Angiogenesis}

Even besides DNA damage, ROS are heavily involved in other key steps of cancer development. For one, they can activate transcription factors like $\mathrm{NF}-\mathrm{\kappa B}$ by destroying the inhibitory unit I $\mathrm{KB} \alpha$ or indirectly through TNF and IL-1, which regulate inflammation. ROS can also regulate and increase the amount of inflammatory cytokines like TNF- $\alpha$ and various interleukins and chemokines, thus inducing chronic inflammation [9]. While occasional inflammation is beneficial and necessary to counteract pathogens, chronic inflammation puts the body at risk for several diseases and cancers, and the role of ROS in it shows yet another link of it to cancer. However, it must be noted that ROS's role in chronic inflammation may not always be harmful. For example, when TNF was blocked in some patients, their chance of developing a lymphoma increased; thus, the relationship is still not clear [13]. To further support this, when ROS activate NF-kB, its activation 
seems to boost cancer development. However, its suppression is also linked to carcinogenesis [14]. Since ROS have so many functions, while they can lead to inflammation, inflammatory cells may also result in more ROS production and a higher risk of developing cancer. This is supported by the fact that tumor promotion is inhibited when phagocyte respiratory bursts (release of large quantities of ROS) are prevented by the use of antioxidants, steroids, and retinoids $[15,16]$.

ROS are critical to tumor cell survival because of their role in signal transduction pathways. One significant example of this is how ROS activate Akt, a serine-threonine kinase whose main functions can be summarized as promote tumor cell survival and suppress antioxidant activity [17]. It inhibits apoptosis by deactivating caspase-9 and Bcl-2-associated death promoter, both of which promote apoptosis in a tumor [9]. Through another pathway, Akt also enhances glucose uptake and glycolysis, which may, but not always, increases the amount of ROS produced in the electron transport chain [18]. In addition, NOX enzymes both produce ROS and enhance a tumor's survival chances [19].

Although the link between ROS and tumor proliferation is not extremely well-studied, it has been demonstrated multiple times that moderate levels of ROS lead to increased cell proliferation. Under conditions of oxidative stress and particularly when hydrogen peroxide is present, various proteins, including the extracellular signal-regulated kinase (ERK) subfamily, Akt, and the p38 subfamily are activated [20]. Through the ERK pathway, various transcription factors involved in the cell cycle are activated and the tumor cells can rapidly progress through it. One of these transcription factors is C-myc which is responsible for the G1/S phase transition and can be looked to as an indicator of cell proliferation because of its upregulation of cyclins [9]. Through c-Jun N-terminal kinase (JNK) and p38 proteins, hydrogen peroxide is able to activate Activator Protein-1 (AP-1) which regulates several aspects of cell proliferation [21]. As stated previously, ROS activates NF- $\kappa B$, which in turn stimulates not only inflammation but also cell proliferation, which is well expressed in many cancerous cells [9]. However, the exact relationship here is also confusing. While moderate levels of ROS stimulate NF- $\kappa \mathrm{B}$ activation, high levels inhibit it, leading to the prevention of cell proliferation [9]. ROS have proven to be important, at least in low to medium levels, in lung cancer cell proliferation, and its inhibition generally also inhibits proliferation [22].

Finally, ROS play a crucial role in both metastasis, when the cancer spreads and a tumor forms in a secondary location, and angiogenesis, the production of blood vessels for the tumor. Regarding metastasis, ROS interacts with a variety of pathways that interact with the cytoskeleton and extracellular matrix. Matrix metalloproteinases (MMPs), a key group of enzymes that sever the movement-inhibiting proteins that suppress metastasis, seem to generally be active under oxidative stress. In particular, MMP-3, MMP-10, and MMP-13 are upregulated under oxidative conditions, and MMP-2 and MMP-9 were activated post transcriptionally under oxidative conditions, especially in the presence of hydrogen peroxide $[23,24]$. This may occur by reacting with the thiol group in the catalytic domain of the inactive MMP [25]. Through the activation of NF- $\kappa$ B by ROS, a signaling pathway results in BLT2 promoting metastasis, and this pathway is also connected to Rasinduced metastasis [26,27]. Thus, ROS seems to be implicated in metastasis through a multitude of pathways summarized in Figure 2. In fact, an experiment established a causal relationship between ROS and metastasis by transferring mitochondrial DNA that was linked to high ROS production to a tumor in mice with traditionally poor metastatic potential [28]. Subsequently, the cells developed a high metastatic potential, and this phenomenon was reversed with the addition of ROS scavengers [28].

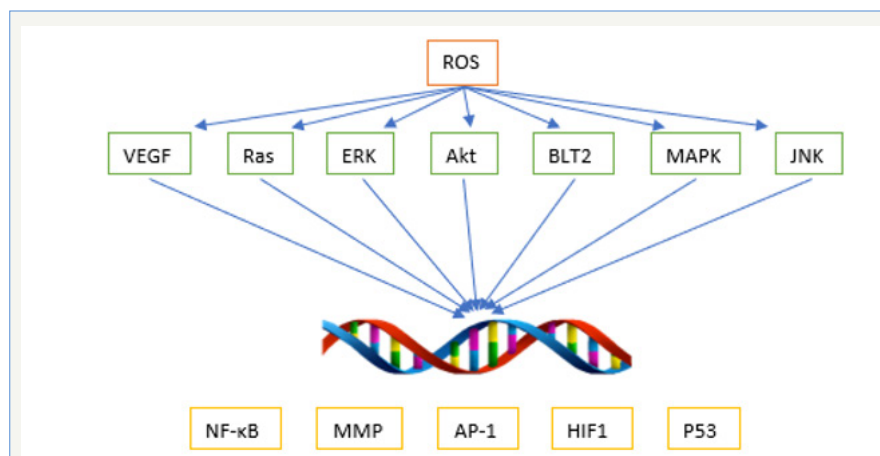

Figure 2: How ROS impacts cancer development through signaling pathways.

Tumors require a surplus of oxygen and nutrients to support their rapid proliferation. In order to get access to this, new blood vessels must form around them. Among the most important proteins for angiogenesis are the vascular endothelial growth factor (VEGF) and the fibroblast growth factor (FGF). Both of these and more, such as the transcription factor HIF- $1 \alpha$, are dependent on ROS production. The growth factors are released in response to certain levels of ROS. This is because ROS production increases under hypoxic conditions, indicating that growth factors are needed to be released in order to provide the cells with oxygen [29]. As additional support, a study found that prostate tumors expressing the Nox family of NADPH oxidases (the one associated with endogenous ROS production) demonstrated an upregulation of VEGF and MMPs. In addition, the effect was reversed by the addition of catalase, indicating the importance of ROS, particularly hydrogen peroxide, in VEGF expression [30]The trend so far seems to be that while low to medium levels of ROS generally promote metastasis and angiogenesis, high levels do just the opposite by suppressing tumor growth.

\section{Treatment Possibilities}

The role of ROS in cancer initiation and development offers 2 main avenues of cancer treatment possibilities. The first is through antioxidants, the natural enemy of ROS. However, several cancer treatments actually involve increasing ROS production.

Antioxidants are a diverse array of molecules, both enzymatic and nonenzymatic, that can be found in the extracellular matrix, mitochondria, and cytoplasm of the cell. The nonenzymatic antioxidants often work by reducing the ROS and later being reduced back to a functional state. Vitamins $C$ (ascorbic acid) and E, specifically $\alpha$-Tocopherol, are perhaps the two most well-known nonenzymatic antioxidants. Both vitamins work by reducing free 
radicals. Vitamin C scavenges ROS while vitamin E scavenges peroxyl radicals, stopping a chain of lipid peroxidation on a cell membrane [1]. In addition, vitamin $\mathrm{C}$ converts vitamin $\mathrm{E}$ free radicals back into functional vitamin E. Another important molecule is a thiol called Glutathione (GSH) which through glutathione peroxidase (GSHPx) reduces hydrogen peroxide by its conversion to water and oxygen [1]. In the process, GSH is oxidized and disulfide bonded with another GSH molecule to form glutathione disulfide (GSSG), which is then reduced back into functional GSH by glutathione reductase, using NADPH as the electron donor [31]. Uric acid is a prominent but less studied antioxidant that also works by reducing ROS like singlet oxygen, hydroxyl radicals, and hydrogen peroxide with an effectiveness comparable to vitamin A [32]. $\beta$-carotene, a carotenoid, is obtained from plants and reacts with both hydroxyl radicals and superoxide anions [33]. Interestingly, $\beta$-carotene's antioxidant effects may be reversed in high oxygen concentrations [34]. Bilirubin, formed from heme metabolism, also reduces free radicals, especially peroxyl radicals, and is converted to biliverdin before being reduced back to bilirubin by biliverdin reductase [35]. It has been found to work complementarily with GSH, as bilirubin tends to be protected against lipid peroxidation while GSH protects against water-soluble protein oxidation, and the concentration of each is determined relative to each other by the environment in the cell [35]. Melatonin is a more unusual antioxidant. Unlike others, it does not neutralize ROS through redox cycling but instead rearranges its molecular structure to accept hydroxyl radicals and hydrogen peroxide [36]. Not only that but melatonin activates enzymatic antioxidants and increases the efficiency of and thus the safety of the electron transport chain. In this way, it indirectly reduces the amount of ROS [37].

There are three major families of enzymatic antioxidants: superoxide dismutase (SOD), catalase, and glutathione peroxidase (GSH-Px). SODs catalyze the conversion of superoxide anions to hydrogen peroxide. There are three forms of SOD: SOD1, which uses copper and zinc as the electron donor and is present in the cytoplasm, SOD2, which uses manganese and is present in the mitochondria, and SOD3 which also uses copper and zinc and is present in the extracellular matrix [38]. Catalase, which uses NADPH as the electron donor, catalyzes the conversion of hydrogen peroxide into water and oxygen. The GSH-Px family catalyzes the conversion of hydrogen peroxide to water using glutathione instead of catalase. There are 4 main forms of GSH-Px known as GSH-Px1-4; each performs a generally similar function, but they prefer different substrates and are located in different places. GSH-Px1 is the most proliferous of them and prefers hydrogen peroxide [39].

With all that being said, while antioxidants clearly protect the cell from ROS and oxidative stress, their use as a treatment is actually very limited. Several clinical studies found that antioxidant supplementation either had no effect on a cancer's incidence and progression or even worsened the chance of developing cancer $[40,41,42]$. Particularly, one study found that $\beta$-carotene and vitamin A supplementation increased the risk of developing lung cancer and had no effect on developing prostate cancer [43]. Still, sometimes supplementation with caution is encouraged. The unfortunate failure of antioxidants to generally be an effective preventative method and treatment leads to the second method of ROS-mediated cancer treatment, actually using high levels of ROS to inhibit cancer progression.

This method of cancer treatment utilizes the observation that most of ROS's upregulating effects on cell proliferation, metastatic disease, and angiogenesis in tumor cells only work when oxidative stress conditions are mild. As noted previously, many of ROS's effects are reversed at higher concentrations, and so several drugs have already taken advantage of that and have been FDA approved. Generally, these drugs work by increasing ROS concentrations so high that they damage the cell to the point of cell death or trigger apoptosis through a variety of pathways depicted in Figure 3 [44]. Even though many of these drugs are nonspecific, normal cells are not severely affected because their natural ROS levels are already low and an increase in ROS concentration can be dealt with by the antioxidant system. This method is not without its drawbacks though. Higher ROS concentrations may lead to increased cancer proliferation in the time before apoptosis is triggered [45]. In addition, while high levels of ROS usually offer sensitivity of tumor cells to chemotherapy, a study found that extended exposure to these high levels led to drug resistance, although the exact mechanism explaining this is not known [46].

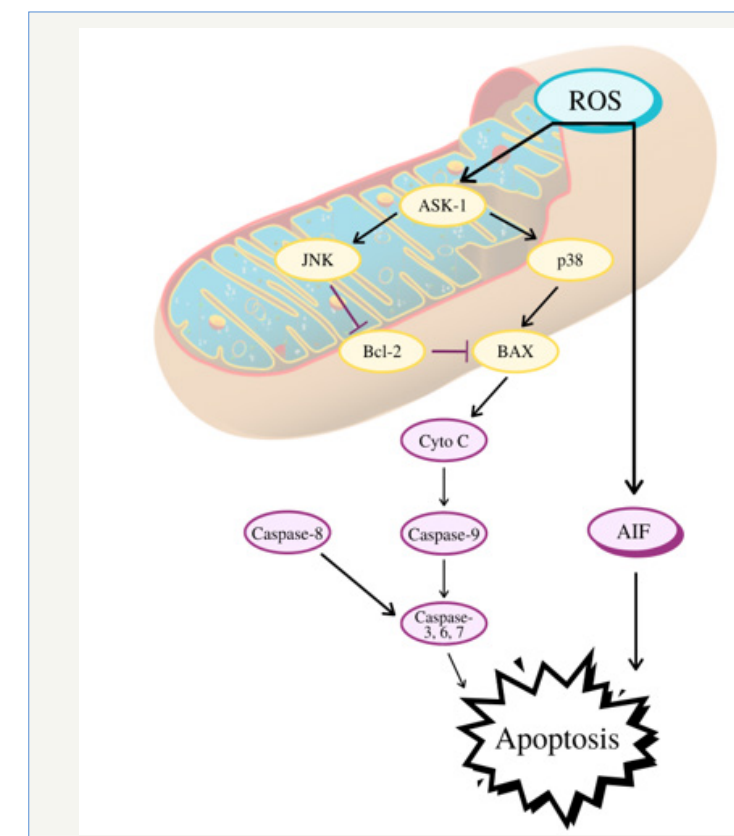

Figure 3: How high ROS levels in cancer cells may lead to apoptosis.

One example is Procarbazine, an early drug which increases hydrogen peroxide production to treat Hodgkin's and nonHodgkin's lymphoma, gliomas, and brain tumors [47]. $\mathrm{As}_{2} \mathrm{O}_{3}$, Elesclomol, Sulindac, Aminoflavone, and many others also work in a similar fashion, by directly stimulating ROS generation [48-51]. Other drugs indirectly stimulate oxidative stress conditions by suppressing antioxidant activity. 2-methoxyestradiol inhibits SODs, and a phase II clinical trial found it to show anticancer activity at a specific concentration [52]. Usage of both L-buthioninesulfoximine, a GSH synthesis inhibitor, and $\mathrm{As}_{2} \mathrm{O}_{3}$ has been found to treat advanced solid tumors [53]. 
ROS can also be used to reduce chemotherapy and radiotherapy resistance in tumor cells. A combination of curcumin and tamoxifen treatment for melanoma resulted in ROS-increase associated apoptosis in the melanoma cells [54]. On top of that, the noncancerous cells were unharmed by the treatment [54]. Another compound, emodin, was found to make gallbladder cancer cells less chemoresistant to the drug cisplatin through ROS generation [55]. For radiotherapy, inhibition of HIF-2 $\alpha$, a protein that mediates the response to hypoxia, was associated with an increase in ROS that ultimately made radiation treatment more effective on lung cancer cells [56].

\section{Conclusion}

Oxidative stress plays an undeniably key role in not only cancer initiation but also proliferation, metastasis, and angiogenesis. ROS can cause direct damage to DNA strands in the form of guanine-thymine transversions, oxidation of bases, strand breaks, substitutions, and more. Luckily, DNA is protected from most hydroxyl radicals, the most dangerous ROS to DNA, by a membrane. However, hydrogen peroxide is more often able to cross the nuclear membrane. Through more indirect pathways like promotion of methyltransferase expression or p62 accumulation, ROS contribute to developing conditions suitable for carcinogenesis. Once a tumor has formed, ROS in low to mid concentrations are usually used to the benefit of tumor cells. They are involved in stimulating chronic inflammation through NF- $\mathrm{KB}$ and TNF. They act through various signaling pathways, such as through Akt, that inhibit antioxidant activity, suppress apoptosis, and generally help the tumor to survive. Perhaps more crucially, ROS play a major function in cell proliferation through, for example, the ERK pathway. Additionally, they can operate through MMPs and the BLT2 pathway to promote metastasis, and through VEGF, FGF, and HIF-1 $\alpha$ to promote angiogenesis.

With ROS playing such a significant role, it might seem as though antioxidants, their natural enemy, might provide a benefit in cancer treatment. However, in essence, the damage has already been done by ROS, so antioxidant-based treatments have generally been found to be ineffective, if not detrimental, in clinical trials. On the other hand, several drugs, both already FDA approved and in current clinical trials, utilize the fact that high ROS concentrations can trigger apoptosis in cancerous cells or otherwise make the cell more responsive to chemotherapy and radiotherapy treatment. Studies have shown that non-cancerous cells are not significantly impacted by this ROS-inducing treatment, likely because their antioxidant system can suppress small increases in ROS concentrations. Current research is heavily focused on this application of ROS, and it should continue to be successful. Since there are so many reactive oxygen species, each affecting unique cellular components, future work needs to be done to understand the entire range of ROS influence in the cell so that more effective treatments can be developed.

\section{References}

1. Birben E, Sahiner UM, Sackesen C, Erzurum S, Kalayci O (2012) Oxidative stress and antioxidant defense. The World Allergy Organization Journal 5(1): 9-19.

2. Finkel T (2011) Signal transduction by reactive oxygen species. The Journal of Cell Biology 194(1): 7-15.
3. Havens CG, Ho A, Yoshioka N, Dowdy SF (2006) Regulation of late $G_{1} / S$ phase transition and APCCdh1 by reactive oxygen species. Molecular and Cellular Biology 26(12): 4701-4711.

4. Massaad CA, Klann E (2011) Reactive oxygen species in the regulation of synaptic plasticity and memory. Antioxid Redox Signal 14(10): 20132054.

5. Higinbotham KG, Rice JM, Diwan BA, Kasprzak KS, Reed CD, et al. (1992) GGT to GTT transversions in codon 12 of the K-ras oncogene in rat renal sarcomas induced with nickel subsulfide or nickel subsulfide/iron are consistent with oxidative damage to DNA. Cancer Res 52(17): 4747 4751 .

6. Hollstein M, Sidransky D, Vogelstein B, Harris CC (1991) p53 mutations in human cancers. Science 253(5015): 49-53.

7. Waris G, Ahsan H (2006) Reactive oxygen species: role in the development of cancer and various chronic conditions. Journal of Carcinogenesis 5: 14.

8. Malins DC, Haimanot R (1991) Major alterations in the nucleotide structure of DNA in cancer of the female breast. Cancer Res 51(19): 5430-5432.

9. Reuter S, Gupta SC, Chaturvedi MM, Aggarwal BB (2010) Oxidative stress, inflammation, and cancer: How are they linked? Free Radical Biology \& Medicine 49(11): 1603-1616.

10. Mates JM, Sanchez-Jimenez FM (2000) Role of reactive oxygen species in apoptosis: Implications for cancer therapy. Int J Biochem Cell Biol 32(2):157-170.

11. Mathew R, Karp CM, Beaudoin B, Vuong N, Chen G, et al. (2009) Autophagy suppresses tumorigenesis through elimination of p62. Cell 137(6): 1062-1075.

12. Das PM, Singal R (2004) DNA methylation and cancer. J Clin Oncol 22(22): 4632-4642.

13. Geborek P, Nitelius E, Noltorp S, Petri H, Jacobsson L, et al. (2005) Population based studies of biological antirheumatic drug use in southern Sweden: comparison with pharmaceutical sales. Ann Rheum Dis 64(12): 1805-1807.

14. Gapuzan ME, Yufit PV, Gilmore TD (2002) Immortalized embryonic mouse fibroblasts lacking the RelA subunit of transcription factor NFkappaB have a malignantly transformed phenotype. Oncogene 21(16): 2484-2492.

15. Rosin MP, Saad el Din Zaki S, Ward AJ, Anwar WA (1994) Involvement of inflammatory reactions and elevated cell proliferation in the development of bladder cancer in schistosomiasis patients. Mutat Res 305(2): 283-292.

16. Weitzman SA, Gordon LI (1990) Inflammation and cancer: Role of phagocyte-generated oxidants in carcinogenesis. Blood 76(4): 655-663.

17. Los M, Maddika S, Erb B, Schulze-Osthoff K (2009) Switching Akt: from survival signaling to deadly response. Bioessays 31(5): 492-495.

18. Manning BD, \& Cantley LC (2007) AKT/PKB signaling: Navigating downstream. Cell 129(7): 1261-1274.

19. Kamata T (2009) Roles of Nox1 and other Nox isoforms in cancer development. Cancer Sci 100(8): 1382-1388.

20. Xia Z, Dickens M, Raingeaud J, Davis RJ, Greenberg ME (1995) Opposing effects of ERK and JNK-p38 MAP kinases on apoptosis. Science 270(5240):1326-1331.

21. Chang L, Karin M (2001) Mammalian MAP kinase signalling cascades. Nature 410(6824): 37-40.

22. Na AR, Chung YM, Lee SB, Park SH, Lee MS, et al. (2008) A critical role for Romo1-derived ROS in cell proliferation. Biochem Biophys Res Commun 369(2): 672-678.

23. Mori K, Shibanuma M, Nose K (2004) Invasive potential induced under long-term oxidative stress in mammary epithelial cells. Cancer Res 64(20): 7464-7472. 
24. WestermarckJ, Kahari VM (1999) Regulation of matrix metalloproteinase expression in tumor invasion. FASEB J 13(8):781-792.

25. Rajagopalan S, Meng XP, Ramasamy S, Harrison DG, Galis ZS (1996) Reactive oxygen species produced by macrophage-derived foam cells regulate the activity of vascular matrix metalloproteinases in vitro. Implications for atherosclerotic plaque stability. J Clin Invest 98(11): 2572-2579.

26. Kim EY, Seo JM, Kim C, Lee JE, Lee KM, et al. (2010) BLT2 promotes the invasion and metastasis of aggressive bladder cancer cells through a reactive oxygen species-linked pathway. Free Radic Biol Med 49(6):10721081.

27. Kim EY, Seo JM, Cho KJ, Kim JH (2010) Ras-induced invasion and metastasis are regulated by a leukotriene B4 receptor BLT2-linked pathway. Oncogene 29(8): 1167-1178.

28. Ishikawa K, Takenaga $K$, Akimoto $M$, Koshikawa N, Yamaguchi A, et al. (2008) ROS-generating mitochondrial DNA mutations can regulate tumor cell metastasis. Science 320(5876): 661-664.

29. Chandel NS, Maltepe E, Goldwasser E, Mathieu CE, Simon MC, et al. (1998) Mitochondrial reactive oxygen species trigger hypoxia-induced transcription. Proceedings of the National Academy of Sciences of the United States of America 95(20): 11715-11720.

30. Arbiser JL, Petros J, Klafter R, Govindajaran B, McLaughlin ER, et al. (2002) Reactive oxygen generated by Nox1 triggers the angiogenic switch. Proc Natl Acad Sci U S A 99(2): 715-720.

31. Forman HJ, Zhang H, Rinna A (2009) Glutathione: Overview of its protective roles, measurement, and biosynthesis. Molecular Aspects of Medicine 30(1-2): 1-12.

32. Ames BN, Cathcart R, Schwiers E, Hochstein P (1981) Uric acid provides an antioxidant defense in humans against oxidant- and radical-caused aging and cancer: A hypothesis. Proceedings of the National Academy of Sciences of the United States of America 78(11): 6858-6862.

33. El-Agamey A, Lowe GM, McGarvey DJ, Mortensen A, Phillip DM, et al (2004) Carotenoid radical chemistry and antioxidant/pro-oxidant properties. Arch Biochem Biophys 430(1): 37-48.

34. Rice-Evans CA, Sampson J, Bramley PM, Holloway DE (1997) Why do we expect carotenoids to be antioxidants in vivo? Free Radic Res 26(4): 381398.

35. Sedlak T, Saleh M, Higginson D, Paul B, Juluri K, et al. (2009) Bilirubin and glutathione have complementary antioxidant and cytoprotective roles Proceedings of The National Academy of Sciences 106(13): 5171-5176.

36. Hacisevki A, Baba B (2018) An overview of melatonin as an antioxidant molecule: A biochemical approach. Intech Open.

37. Reiter RJ, Tan D, Mayo JC, Sainz RM, Leon J, et al. (2003) Melatonin as an antioxidant: Biochemical mechanisms and pathophysiological implications in humans. Acta Biochimica Polonica 50(4): 1129-1146.

38. Fukai T, Ushio-Fukai M (2011) Superoxide dismutases: role in redox signaling, vascular function, and diseases. Antioxidants \& redox signaling 15(6): 1583-1606.

39. Lubos E, Loscalzo J, Handy DE (2011) Glutathione peroxidase-1 in health and disease: From molecular mechanisms to therapeutic opportunities. Antioxidants \& redox signaling 15(7): 1957-1997.

40. Blot WJ, Li JY, Taylor PR, et al. (1993) Nutrition intervention trials in Linxian, China: supplementation with specific vitamin/mineral combinations, cancer incidence, and disease-specific mortality in the general population. Journal of the National Cancer Institute 85(18): 1483-1491.
41. Hennekens CH, Buring JE, Manson JE, Stampfer M, Rosner B, et al. (1996) Lack of effect of long-term supplementation with beta carotene on the incidence of malignant neoplasms and cardiovascular disease. New England Journal of Medicine 334(18): 1145-1149.

42. Lee IM, Cook NR, Manson JE (1999) Beta-carotene supplementation and incidence of cancer and cardiovascular disease: Women's Health Study. Journal of the National Cancer Institute 91(24): 2102-2106.

43. Omenn GS, Goodman GE, Thornquist MD, Balmes J, Cullen MR, et al. (1996) Effects of a combination of beta carotene and vitamin A on lung cancer and cardiovascular disease. New England Journal of Medicine 334(18): 1150-1155.

44. Liou GY, Storz P (2010) Reactive oxygen species in cancer. Free radical research 44(5): 479-496.

45. Trachootham D, Alexandre J, Huang P (2009) Targeting cancer cells by ROS-mediated mechanisms: A radical therapeutic approach? Nat Rev Drug Discov 8(7): 579-591.

46. Maiti AK (2010) Gene network analysis of oxidative stress-mediated drug sensitivity in resistant ovarian carcinoma cells. Pharmacogenomics J10(2): 94-104.

47. Berneis K, Bollag W, Kofler M, Luthy H (1966) The enhancement of the after effect of ionizing radiation by a cytotoxic methylhydrazine derivative. Eur J Cancer 2(1): 43-49.

48. Jing Y, Dai J, Chalmers-Redman RM, Tatton WG, Waxman S (1999) Arsenic trioxide selectively induces acute promyelocytic leukemia cell apoptosis via a hydrogen peroxide-dependent pathway. Blood 94(6): 2102-2111.

49. Kirshner JR, He S, Balasubramanyam V, Kepros J, Yang CY, et al. (2008) Elesclomol induces cancer cell apoptosis through oxidative stress. Mol Cancer Ther 7(8): 2319-2327.

50. Marchetti M, Resnick L, Gamliel E, Kesaraju S, Weissbach H, et al. (2009) Sulindac enhances the killing of cancer cells exposed to oxidative stress. PLoS One 4(6): e5804.

51. McLean L, Soto U, Agama K, Francis J, Jimenez R, etal. (2008) Aminoflavone induces oxidative DNA damage and reactive oxidative species-mediated apoptosis in breast cancer cells. Int J Cancer 122(7):1665-1674.

52. Sweeney C, Liu G, Yiannoutsos C, Kolesar J, Horvath D, et al. (2005) A phase II multicenter, randomized, double-blind, safety trial assessing the pharmacokinetics, pharmacodynamics, and efficacy of oral 2-methoxyestradiol capsules in hormone-refractory prostate cancer. Clin Cancer Res 11(18): 6625-6633.

53. Maeda H, Hori S, Ohizumi H, Segawa T, Kakehi Y, et al. (2004) Effective treatment of advanced solid tumors by the combination of arsenic trioxide and L-buthionine-sulfoximine. Cell Death Differ 11(7): 737-746.

54. Chatterjee SJ, Pandey S (2011) Chemo-resistant melanoma sensitized by tamoxifen to low dose curcumin treatment through induction of apoptosis and autophagy. Cancer Biol Ther 11(2): 216-228.

55. Wang W, Sun Y, Li X, Li H, Chen Y, et al. (2011) Emodin potentiates the anticancer effect of cisplatin on gallbladder cancer cells through the generation of reactive oxygen species and the inhibition of survivin expression. Oncol Rep 26(5): 1143-1148.

56. Bertout JA, Majmundar AJ, Gordan JD, Lam JC, Ditsworth D, et al. (2009) HIF2alpha inhibition promotes p53 pathway activity, tumor cell death, and radiation responses. Proceedings of the National Academy of Sciences of the United States of America 106(34): 14391-14396. 